\title{
Towards the first radio galaxies
}

\author{
Israel Matute 1,2 (1), Jose Afonso ${ }^{1,2}$, Luca Bizzocchi ${ }^{3}$, Cirino \\ Pappalardo $^{1,2}\left(\mathbb{0}\right.$, Hugo Messias ${ }^{4}$ and Stergios Amarantidis ${ }^{1,2}$ \\ ${ }^{1}$ Institute of Astrophysics and Space Sciences, Lisbon, PT \\ email: imatute@oal.ul.pt \\ ${ }^{2}$ Faculty of Sciences, Univ. of Lisbon, Lisbon, PT \\ ${ }^{3}$ Max Planck Institute for Extraterrestrial Physics (MPE), Garching, DE \\ ${ }^{4}$ Joint ALMA Observatory, Santiago de Chile, CHL
}

\begin{abstract}
Powerful AGN have been detected up to very high redshifts $(\mathrm{z} \sim 6-8)$, well within the Epoch of Reionization (EoR), but the lack of powerful radio-galaxies among such sources strongly disagrees with the expectations based on the known radio population up to $\mathrm{z} \sim 5$. Our group has been pursuing a detailed analysis of the faintest population of radio sources detected in the deepest fields searching for clues of these first radio galaxies. This paper describes our strategy and presents a highly confident candidate. The results, once follow-up of all candidates is completed, will have significant implications for the upcoming generation of all-sky deep radio surveys such as ASKAP-EMU, Westerbork-WODAN, and SKA itself.
\end{abstract}

Keywords. galaxies: active, galaxies: high-redshift, galaxies: distances and redshifts, galaxies: evolution, radio continuum: galaxies, submillimeter

\section{Overview}

One of the most fundamental objectives of extragalactic astronomy is the understanding of the birth of the first objects in the Universe, and how this led an almost completely neutral Universe, at redshifts $z \gtrsim 11$, to an almost completely ionised one at redshifts of around 6-7. Although theory has advanced significantly over the last few years (Santos et al. 2011), direct observations of sources in the Epoch of Reionisation (EoR) are still only barely manageable: distant powerful quasars, at $z \sim 6-8$, and star-forming galaxies selected from Lyman-break techniques possibly reaching $z \sim 8-11$ (Oesch et al. 2016); and the occasional detection of elusive gamma-ray burst hosts.

Furthermore, the statistical detection of neutral hydrogen will be performed with LOFAR and MWA beyond $z \sim 6$ while in the next decade, the SKA will be able to directly study the HI $21 \mathrm{~cm}$ forest against a bright Radio Galaxy (RG), placed well within the EoR - this relies, however, in finding even more distant powerful RGs.

But, even though $i$ ) current radio telescopes are sensible enough to detect equivalent sources to local powerful RGs (like 3C273 and Cygnus A) up to z $~ 7$; ii) QSOs have been detected up to $\mathrm{z} \sim 7.5$ (Bañados et al. 2018); iii) cosmological simulations show that even earlier activity is possible within the $\Lambda$ CDM framework through hierarchical, gasrich mergers and $i v$ ) the extrapolation of radio LFs predict a significant number of RGs above $z>6$ (30 radio-loud AGN per $\operatorname{deg}^{2}$ at $100 \mu \mathrm{Jy}$ flux level; $\mathrm{S}^{3}$ simulations, Wilman et al. (2008)), only a handful have been detected so far at $z \sim 6$ (Fig. 1).

\section{Methodology and current status}

Our group has been exploring the possibility that many of these expected high-z RGs are hidden within the faint, unidentified fraction of radio sources in the deep cosmological 

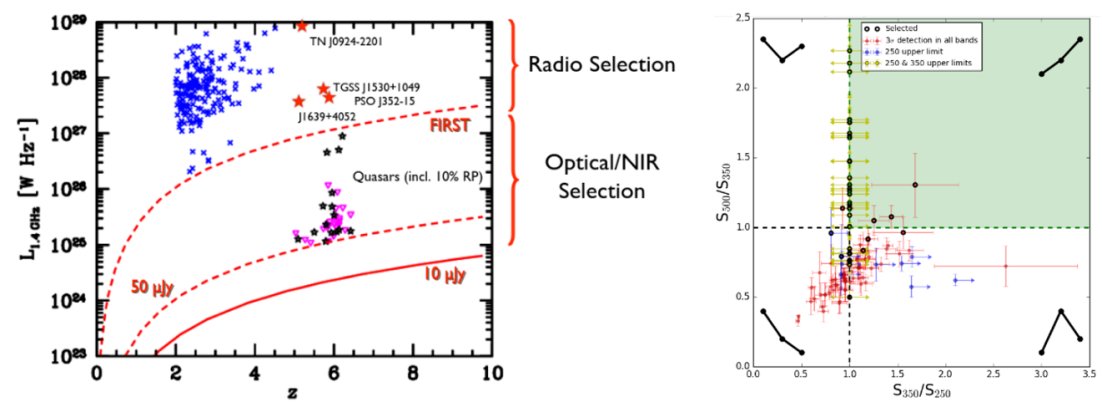

Figure 1. Left) Compilation of the highest-z known Radio-Galaxies . Right) Herschel colors for radio candidates in the COSMOS field were used as proxies for their high-z nature.
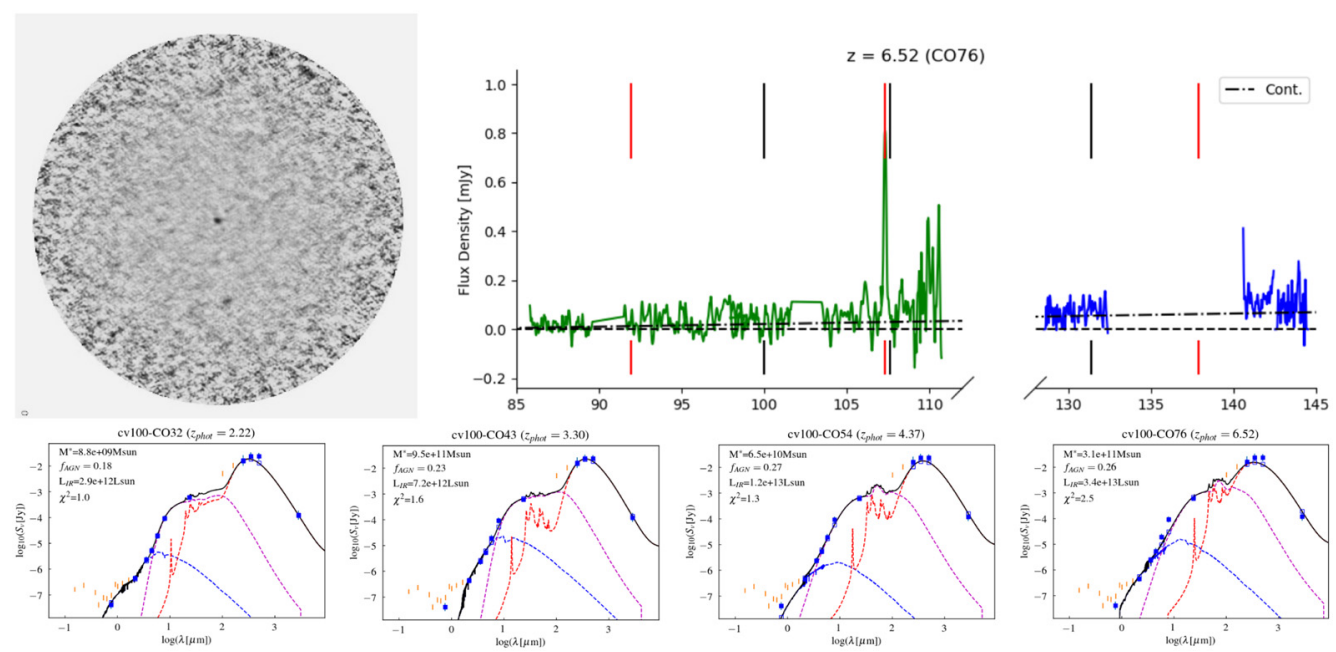

Figure 2. Top-left) ALMA 3mm continuum image of CVLA100. Top-right) Combined band3 + band4 spectrum of CVLA100 showing only one single line at the 10- $\sigma$ level. The continuum level, indicated by the dot-dashed line, spectral index is compatible con thermal emission in the Rayleigh-Jeans regime $(\alpha \sim 1.9 \pm 0.2)$. Bottom $)$ The best fits are found at the redshift permitted by the line detection and have been computed using SED3Fit Berta et al. (2013).

fields. We use the FIR colors (Fig. 1) as a proxy of their high-z nature (Dowell et al. 2014) and measured the Herschel SPIRE fluxes using the full timeline information at radio position (Bendo et al. 2013). The candidates are selected after a detailed analysis of the photo-z based on their UV-to-radio SED. A handful of candidates have been found in the COSMOS + ECDFS fields while analysis is ongoing in other fields (UDS, AEGIS, GAMA, Stripe82).

One of the most promising candidates is CVLA100. Its overall SED is shown in Fig. 2 as well as its mm spectrum after follow-up with IRAM and ALMA. Although the redshift of the source is still not constrained due to the detection of a single line in the ALMA spectrum, the possibilities are severely limited and are currently being extensively evaluated by a detailed analysis with energy balance algorithms such as SED3FIT and CIGALE. The conclusions of this work, whether the high-z nature of this (or any other) candidate source is confirmed or not, will impact the definition of future radio surveys with SKA. 


\section{References}

Banados, E., Venemans, B. P., \& Mazzucchelli, C. 2018, Nature, 553, 473B

Bendo, G. et al. 2013, MNRAS, 433, 3062

Berta, S., Lutz, D., Santini, P., et al. 2013, A\&A A, 551, 100

Dowell, C. D., Conley, A., Glenn, J., et al. 2014, ApJ, 780, 75

Oesch, P. A., Brammer, G., van Dokkum, P. G., et al. 2016, ApJ, 819, 2

Santos, M. G., Silva, M. B., Pritchard, J. R., Cen, R., \& Cooray, A. 2011, A\&3A, 527, A93

Wilman, R. J., Miller, L., Jarvis, M. J., et al. 2008, MNRAS, 388, 1335 